\title{
Effects of glycyrrhizin on the differentiation of myeloid cells of the heart and lungs in lipopolysaccharide-induced septic mice
}

\author{
Eun-Hye Seo ${ }^{1}$, Ga-Yun Song ${ }^{2}$, Byung Ok Kwak², Chung-Sik Oh ${ }^{3}$, Ilsoon Son ${ }^{3}$, Seung Hyun
}

Lee $^{2}$, Seong-Hyop Kim ${ }^{3,4}$

\section{Introduction}

Sepsis is a life-threatening systemic immune response and one of the leading causes of morbidity and mortality in the intensive care unit. For the management of sepsis, agents used to control the activation of the immune system are widely applied, including glycyrrhizin (GR).

GR is a bioactive plant component isolated from licorice root with anti-inflammatory activities. The mechanism of its antiinflammatory effect has been determined: it inhibits nitric oxide activity and blocks the activation of high-mobility group box 1 signaling; consequently, it exerts a protective effect on sepsis by inhibiting cytokine production through regulation of the humoral immune response. Cell-mediated immunity is also expected to be involved in this mechanism, but research is limited.

Cluster of differentiation (CD)11b+Gr1 myeloid cells are responsible for cell-mediated immunity. They develop into immune-activated cells such as macrophages, dendritic cells, and neutrophils or immune-suppressive cells such as myeloidderived suppressor cells (MDSCs). The balance between immune-activated cells and MDSCs is believed to be important for protection against multi-organs failure in sepsis.

We hypothesized that the protective effects of GR on sepsis are associated with the regulation of $C D 11 b+G r 1$ myeloid cells. This study investigated the effects of GR on the ratio of MDSCs to $\mathrm{CD} 11 \mathrm{~b}+\mathrm{Gr} 1$ myeloid cells in the heart and lungs in lipopolysaccharide (LPS)-induced septic mice.

\section{Methods}

Mice were divided into three groups (10 per group): Control, LPS, and LPS+GR. After intraperitoneal injection of phosphatebuffered saline for the Control group, LPS for the LPS group, and a combination of LPS and GR for the LPS+GR group, fluorescence-activated cell sorting was utilized to evaluate cytokines and immune cells in the blood, heart, and lungs. Histopathologic analysis of Toll-like receptor (TLR)4 in each organ (10 per group) was also performed.

\section{Results}

The cytokine amounts in the LPS and LPS+GR groups were significantly higher than in the Control group; however, that in the LPS+GR group was significant lower than in the LPS group(Table 1). The ratio of MDSCs to CD11b+Gr1 myeloid cells in the LPS+GR group was significantly higher than in the LPS group but was significantly lower than in the Control group(Fig 1). According to the in vitro assay, The ratio of MDSCs to CD11b+Gr1 myeloid cells in LPS and GR-treated cells was significantly higher compared with pre-culture or LPS treatment (Table 2). The LPS and LPS+GR groups showed significant changes, including inflammatory cell infiltration, widespread alveolar wall thickening, and alveolar hemorrhage on the histopathologic analysis (Figure 2). The staining intensity of TLR4 showed the same pattern as that of cytokines in the heart and lungs. TLR4 staining was significantly lower in the MDscs LPS+GR group than in the LPS group but was higher than that in the Control group(Fig 3).

\section{Conclusion}

GR exhibited protective effects on the heart and lungs in LPSinduced septic mice. The effects were associated with an elevated ratio of MDSCs to CD11b+Gr1 myeloid cells and the inhibition of cytokine release and TLR4 expression after GR injection.
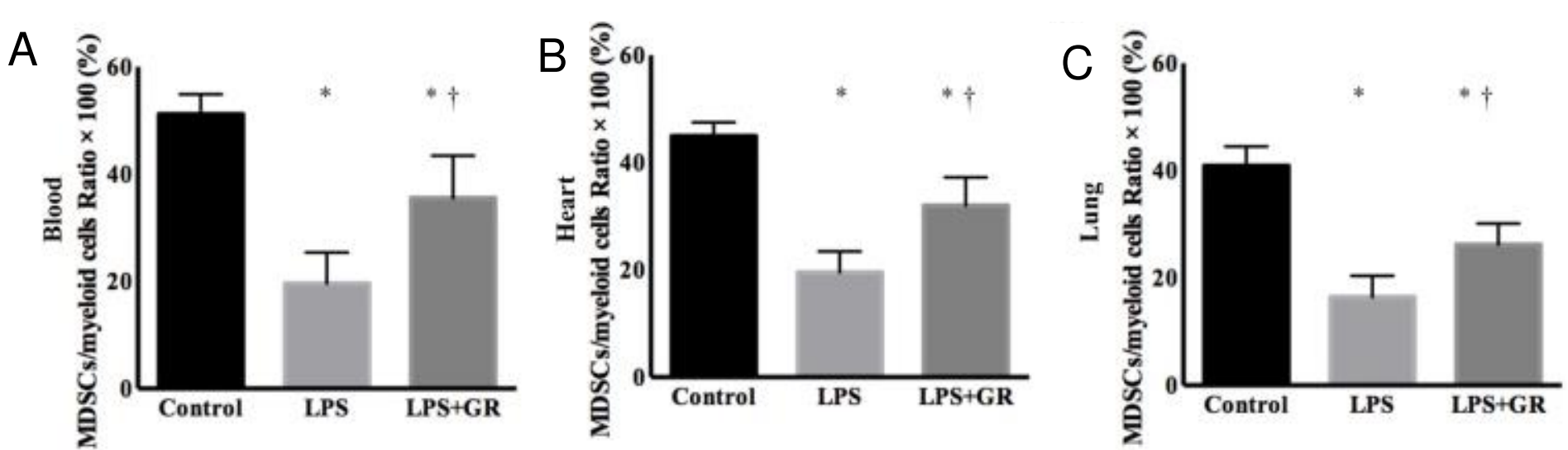

Figure 1. The ratio of myeloid-derived suppressor cells (MDSCs) to myeloid cells $\times 100$ in the blood, heart, and lungs.
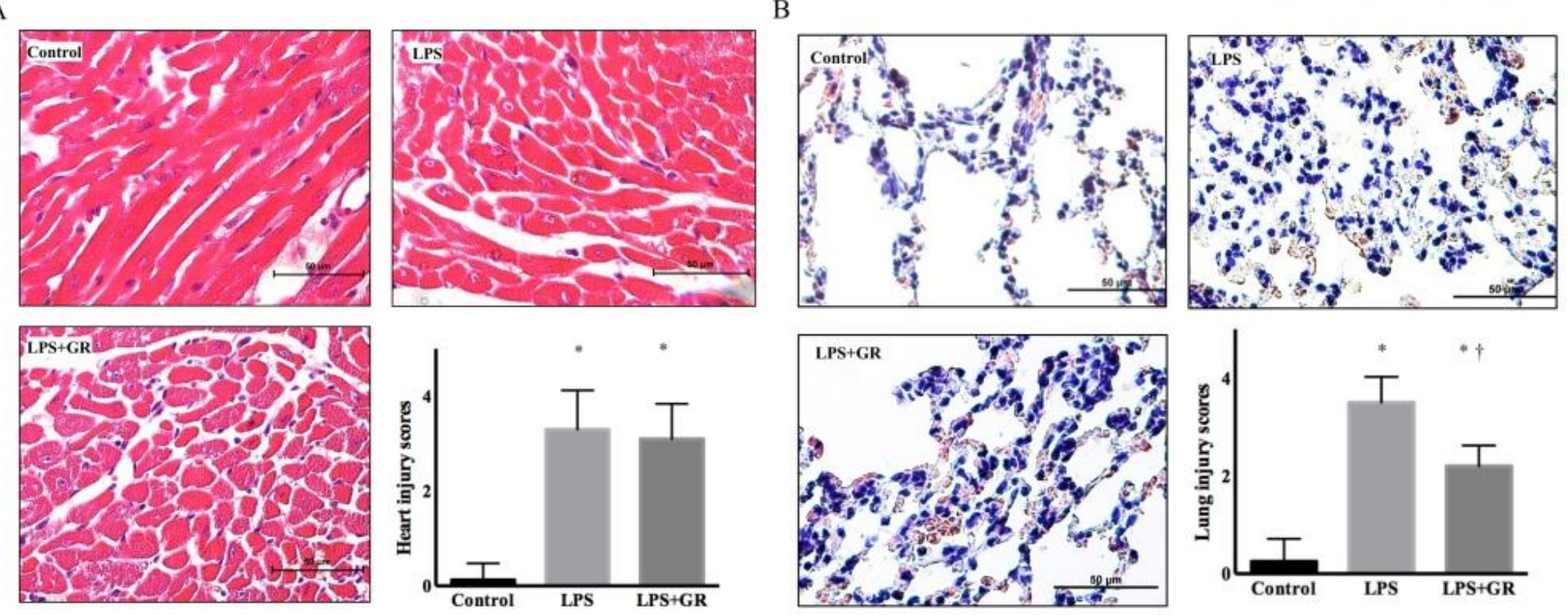

Figure 2. Histopathological findings in the heart $(A)$ and lungs $(B)$.
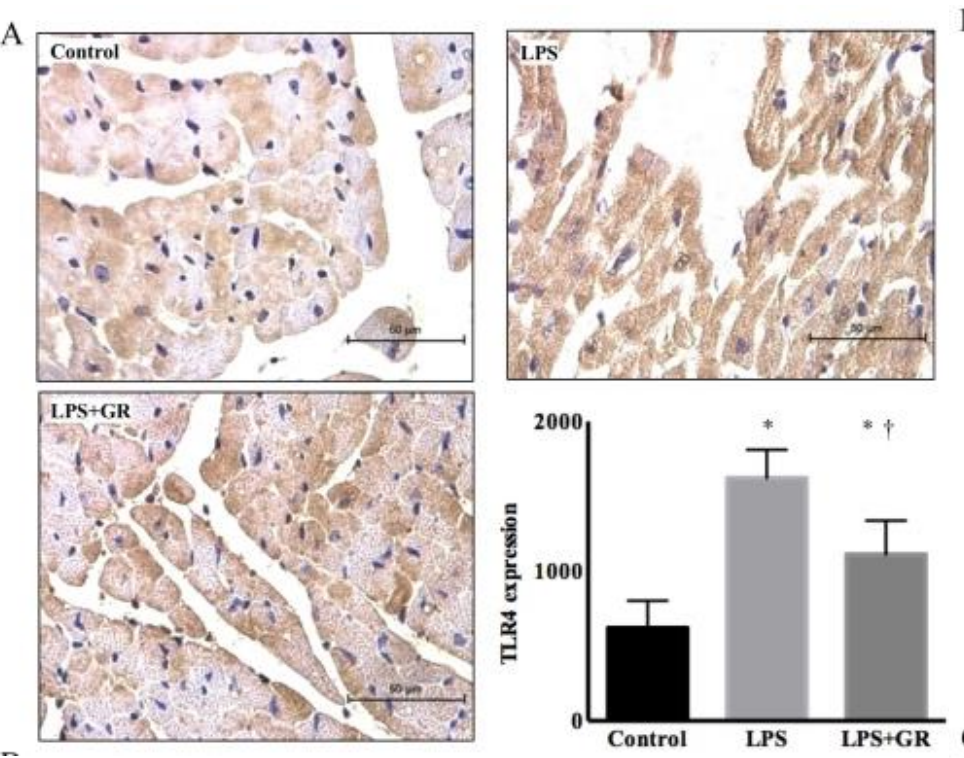

Figure 3. Immunohistochemical staining for Toll-like receptor (TLR) 4 in the heart $(A)$ and lungs $(B)$. Western blot analysis to confirm the intensity of Toll-like receptor (TLR)4 in the heart and lungs $(\mathrm{C})$.

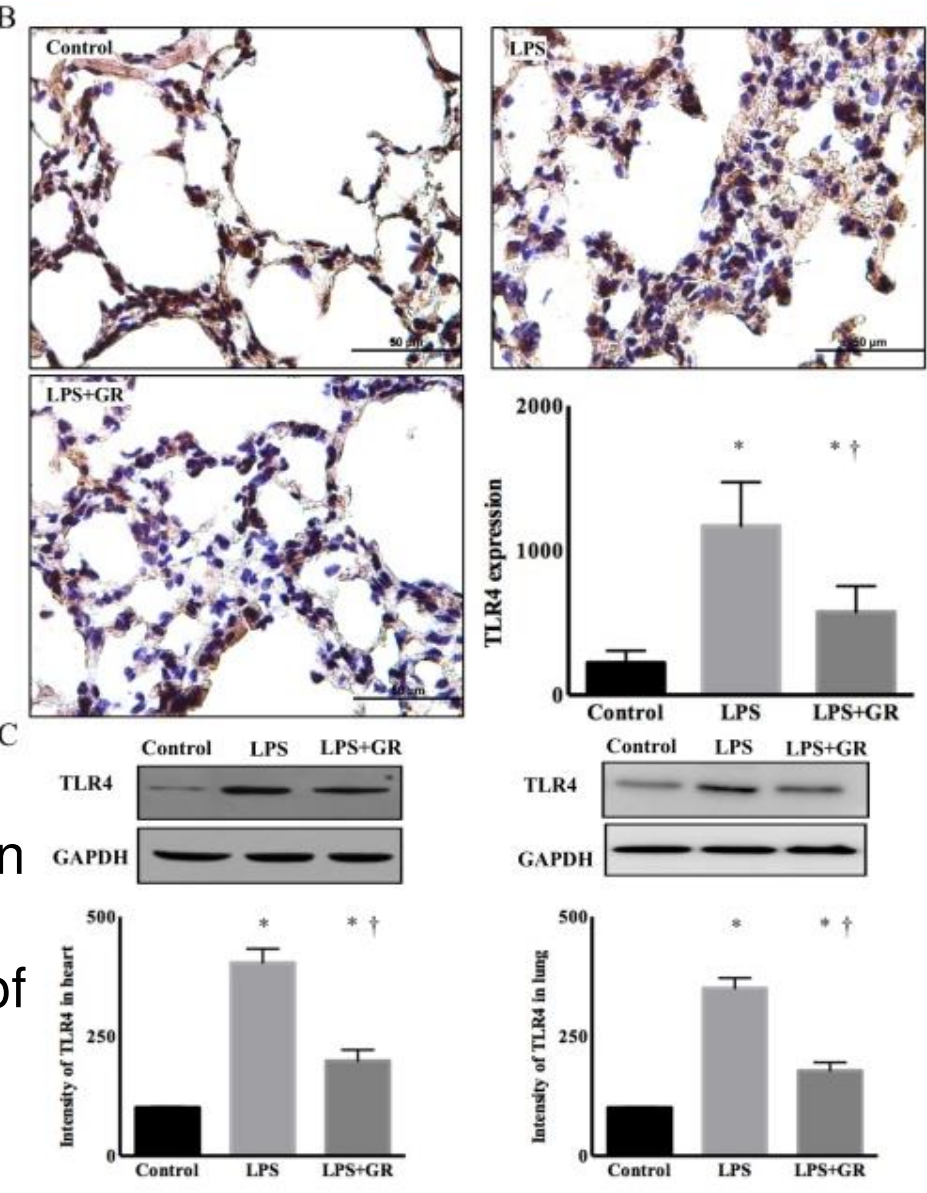

\begin{tabular}{|c|c|c|c|c|c|c|}
\hline & Control group & LPS group & LPS+GR group & Control group & LPS group & LPS+GR group \\
\hline Tumor necrosis factor-alpha $(\mathrm{pg} / \mathrm{mL})$ & $70.11 \pm 21.76$ & $284.20 \pm 56.96^{*}$ & $167.81 \pm 37.27^{* \dagger}+$ & $18.32 \pm 6.64$ & $98.78 \pm 9.17^{*}$ & $49.52 \pm 13.20 *+$ \\
\hline Interleukin-6 (pg/mL) & $11.45 \pm 3.56$ & $650.26 \pm 11.02^{*}$ & $132.66 \pm 13.44^{*}+$ & $36.51 \pm 7.96$ & $172.63 \pm 14.22^{*}$ & $38.16 \pm 12.01 t$ \\
\hline Interleukin-I beta (pg/mL) & $15.52 \pm 2.43$ & $164.82 \pm|7.8|^{*}$ & $34.38 \pm 6.66^{*}+$ & $21.22 \pm 4.23$ & $|26.28 \pm 7.6|^{*}$ & $72.81 \pm 11.02 *$ \\
\hline
\end{tabular}

values are expressed as the mean \pm standard deviation

Table 1. Cytokines from the bronchoalveolar lavage fluid and blood.

$\begin{array}{lll}\text { Control } & \text { LPS } & \text { LPS + GR } \\ 50,000 & 47,300 \pm 1,200 & 48,700 \pm 800\end{array}$

Table 2. In vitro assay on bone marrow. 